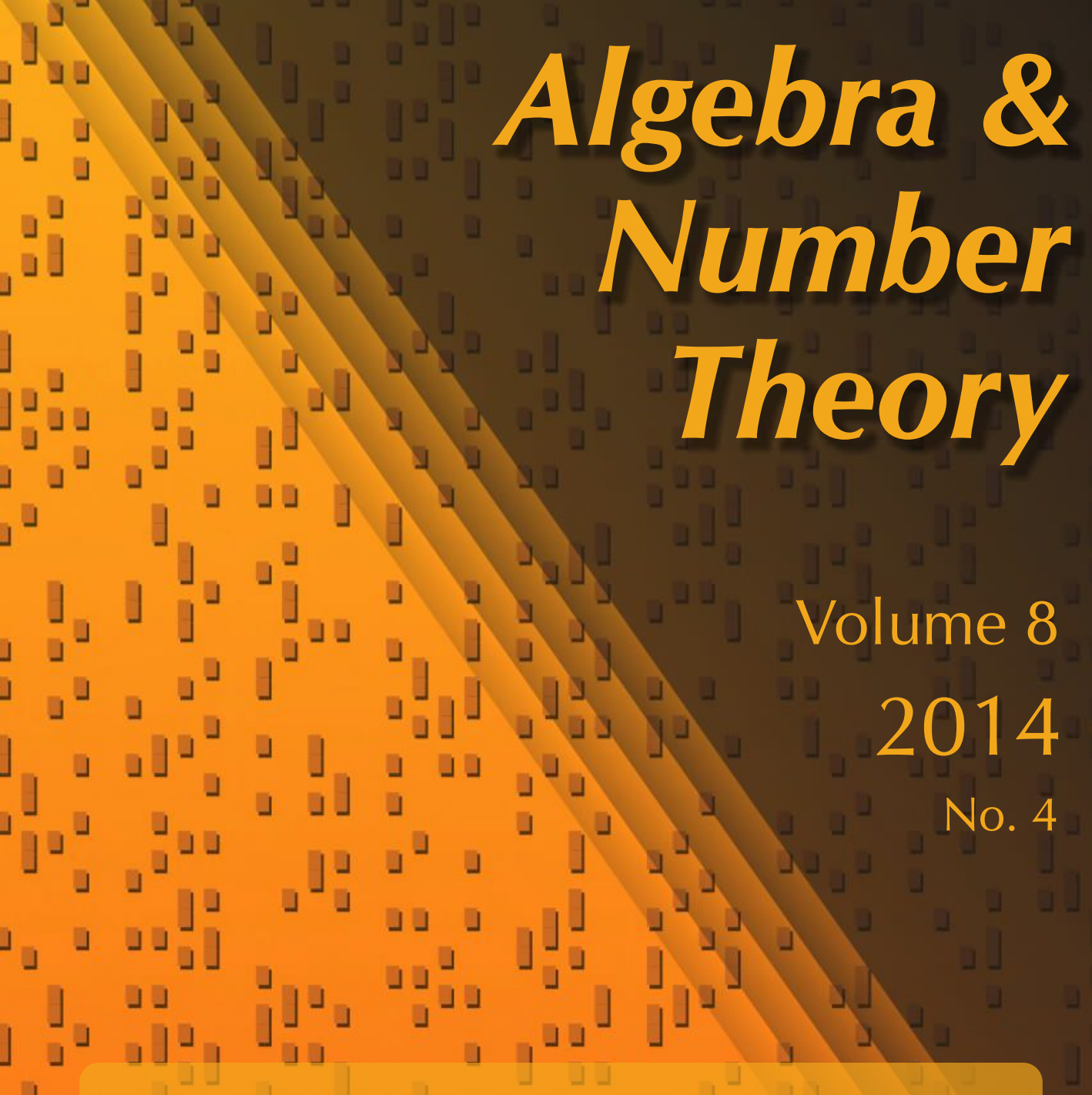

\title{
Étale contractible varieties in positive characteristic
}

Armin Holschbach, Johannes Schmidt and Jakob Stix

\lrcorner

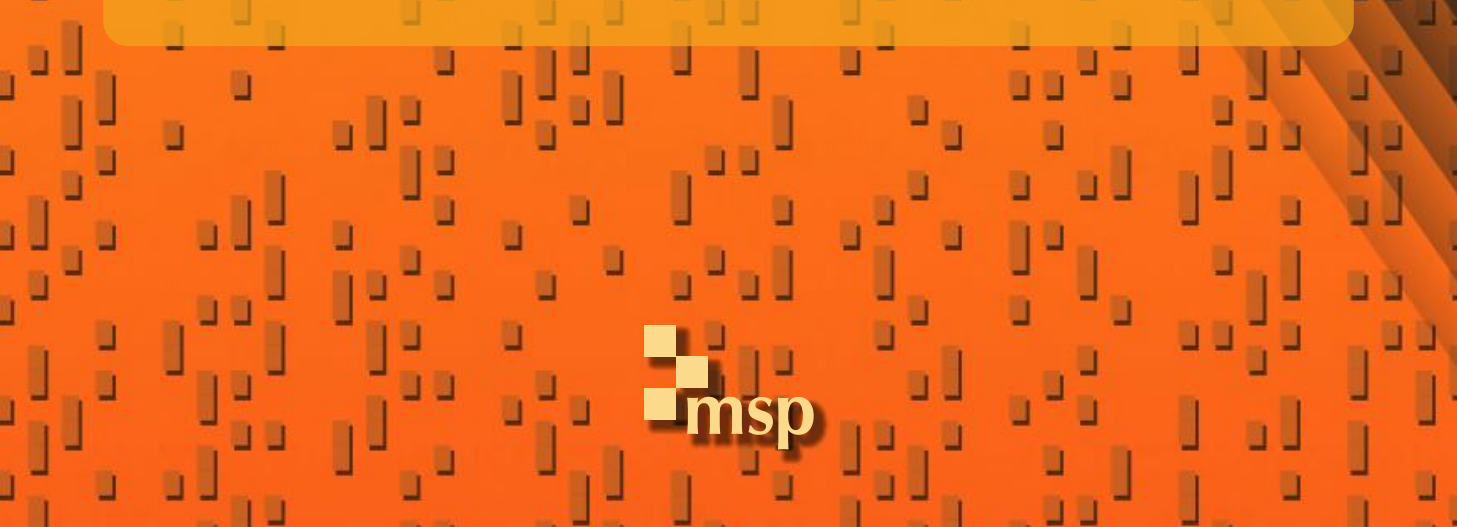




\title{
Étale contractible varieties in positive characteristic
}

\author{
Armin Holschbach, Johannes Schmidt and Jakob Stix
}

Unlike in characteristic 0 , there are no nontrivial smooth varieties over an algebraically closed field $k$ of characteristic $p>0$ that are contractible in the sense of étale homotopy theory.

\section{Introduction}

Homotopy theory is founded on the idea of contracting the interval, either as a space, or as an actual homotopy, that is, a path in a space of maps. In algebraic geometry, the affine line $\mathbb{A}_{k}^{1}$ serves as an algebraic equivalent of the interval, at least in characteristic 0 , where $\mathbb{A}_{k}^{1}$ is contractible.

Matters differ in characteristic $p>0$, where $\pi_{1}\left(\mathbb{A}_{k}^{1}\right)$ is an infinite group: a group $G$ occurs as a finite quotient of $\pi_{1}\left(\mathbb{A}_{k}^{1}\right)$ precisely if $G$ is a quasi- $p$-group due to Abhyankar's conjecture for the affine line as proven by Raynaud. This raises the question whether there is an étale contractible variety in positive characteristic.

Theorem 1. Let $k$ be an algebraically closed field of characteristic $p>0$. A smooth variety $U / k$ is étale contractible if and only if $U=\operatorname{Spec}(k)$ is the point.

It turns out that our discussion in positive characteristic depends only on $\mathrm{H}^{1}$ and $\mathrm{H}^{2}$. By the étale Hurewicz and Whitehead theorems (see [Artin and Mazur 1969, §4]), we might therefore replace "étale contractible" with "étale 2-connected" in Theorem 1. Further, our proof covers more than just smooth varieties. Here is the more precise result, which proves Theorem 1 because smooth varieties have big Cartier divisors.

Theorem 2. Let $k$ be an algebraically closed field of characteristic $p>0$ and let $U / k$ be a normal variety such that

(i) the group $\mathrm{H}_{\mathrm{ett}}^{1}\left(U, \mathbb{F}_{p}\right)$ vanishes,

(ii) there is a prime number $\ell \neq p$ such that $\mathrm{H}_{\mathrm{ét}}^{2}\left(U, \mu_{\ell}\right)=0$,

Supported by DFG-Forschergruppe 1920, "Symmetrie, Geometrie und Arithmetik", HeidelbergDarmstadt.

MSC2010: 14F35.

Keywords: étale homotopy theory, étale contractible. 
(iii) $U$ has a big Cartier divisor or $\operatorname{dim} U \leq 2$.

\section{Then $U$ has dimension 0.}

In order to show the range of varieties to which Theorem 2 applies, we list in Proposition 4 properties of varieties that imply the existence of big Cartier divisors, including quasiprojective varieties and locally $\mathbb{Q}$-factorial (in particular smooth) varieties. The proof of Theorem 2 in the presence of a big Cartier divisor will be given in Section 2.3. The case of normal surfaces will be treated in Section 3.

In the proof of Theorem 2, one would like to work with a compactification $U \subseteq X$ and the geometry of line bundles on $U$ versus $X$. For that strategy to work, we need a compactification that is locally factorial along $Y=X \backslash U$. Since in characteristic $p>0$, resolution of singularities is presently absent in dimension $\geq 4$, we resort to desingularisation by alterations due to de Jong. Unfortunately, the alteration typically destroys the étale contractibility assumption. We first deduce more coherent properties from étale 2-connectedness that transfer to the alteration.

The key difference with characteristic 0 comes from Artin-Schreier theory relating $\mathrm{H}_{\mathrm{ett}}^{1}\left(U, \mathbb{F}_{p}\right)$ to regular functions on $U$.

Remark 3. Let us illustrate the situation in characteristic 0 in contrast to Theorem 1.

(1) There are contractible complex smooth surfaces other than $\mathbb{A}_{\mathbb{C}}^{2}$. The first such example is due to Ramanujam [1971, §3]; see also [tom Dieck and Petrie 1990] for explicit equations. All of them are affine and have rational smooth projective completions.

(2) Smooth varieties $U / \mathbb{C}$ different from affine space $\mathbb{A}_{\mathbb{C}}^{n}$ but with $U(\mathbb{C})$ diffeomorphic to $\mathbb{C}^{n}$ are known as exotic algebraic structures on $\mathbb{C}^{n}$. These varieties are contractible and we recommend the Bourbaki talk on $\mathbb{A}^{n}$ by Kraft [1996], or the survey by Zaĭdenberg [1999]. A remarkable nonaffine (but quasiaffine) example $U$ was obtained by Winkelmann [1990] as a quotient $U=\mathbb{A}^{5} / \mathbb{G}_{\mathrm{a}}$, and more concretely as the complement in a smooth projective quadratic hypersurface in $\mathbb{P}_{\mathbb{C}}^{5}$ of the union of a hyperplane and a smooth surface.

(3) The notion of $\mathbb{A}^{1}$-contractibility is a priori stronger than contractibility in the complex topology. Asok and Doran [2007] construct, for every $d \geq 6$, continuous families of pairwise nonisomorphic, nonaffine smooth varieties of dimension $d$ that are even $\mathbb{A}^{1}$-contractible.

Notation. Throughout the note, $k$ will be an algebraically closed field. By definition, a variety over $k$ is a separated scheme of finite type over $k$. We will denote the étale fundamental group by $\pi_{1}$ and its maximal abelian quotient by $\pi_{1}^{\mathrm{ab}}$. The sheaf $\mu_{\ell}$ for $\ell$ different from the characteristic denotes the (locally) constant sheaf of $\ell$-th roots of unity. 


\section{Big Cartier divisors}

2.1. Existence of big divisors. Recall that a Cartier divisor $D$ on a normal (but not necessarily proper) variety $U / k$ is big if the rational map associated to the linear system $|m D|$ is generically finite for $m \gg 0$.

Proposition 4. Let $k$ be an algebraically closed field and let $U / k$ be a normal variety such that one of the following holds:

(a) $U$ is quasiprojective.

(b) $U$ is a product of varieties with big divisors.

(c) $U$ is locally $\mathbb{Q}$-factorial everywhere.

Then $U$ has a big Cartier divisor.

Proof. Since any ample divisor is big, the conclusion holds if we assume (a). In case (b), the sum of the pullbacks of big Cartier divisors on the factors is again big.

If (c) holds, then we first choose a dense affine open $V \subseteq U$ and an effective big Cartier divisor $D$ on $V$ by (a). Let $B=U \backslash V$ be the boundary, in fact a Weil divisor since $V$ is affine, and let $D^{\prime}$ be the Zariski closure of $D$ as a Weil divisor on $U$. By assumption, $m D^{\prime}$ and $m B$ are both effective Cartier divisors for $m \gg 0$, and there are sections $s_{0}, \ldots, s_{d} \in \mathrm{H}^{0}(V, m D)$ such that the induced map $V \rightarrow \mathbb{P}_{k}^{d}$ is generically finite. For $r \gg 0$, the sections $s_{i}$ extend to sections of

$$
\mathrm{H}^{0}(U, m D+m r B),
$$

so that $m D+m r B$ is the desired big Cartier divisor on $U$.

2.2. Geometry of varieties with vanishing $\mathbf{H}^{1}$ and $\mathbf{H}^{2}$. Let $\ell$ be a prime number different from the characteristic of $k$ and let $U / k$ be a variety with $\mathrm{H}_{\text {et }}^{2}\left(U, \mu_{\ell}\right)=0$. It follows from the Kummer sequence in étale cohomology that $\operatorname{Pic}(U)$ is an $\ell$ divisible abelian group.

The following crucially depends on $k$ being a field of positive characteristic.

Proposition 5. Let $k$ be of characteristic $p>0$. If $U / k$ is a connected reduced variety such that $\pi_{1}^{\mathrm{ab}}(U) \otimes \mathbb{F}_{p}$ is finite, then $\mathrm{H}^{0}\left(U, \mathrm{O}_{U}\right)=k$.

Proof. We argue by contradiction. If $f: U \rightarrow \mathbb{A}_{k}^{1}$ is a dominant map, then the induced map

$$
f_{*}: \pi_{1}^{\mathrm{ab}}(U) \otimes \mathbb{F}_{p} \rightarrow \pi_{1}^{\mathrm{ab}}\left(\mathbb{A}_{k}^{1}\right) \otimes \mathbb{F}_{p}
$$

has image of finite index in the infinite group $\pi_{1}^{\mathrm{ab}}\left(\mathbb{A}_{k}^{1}\right) \otimes \mathbb{F}_{p}$, a contradiction.

By the duality $\mathrm{H}_{\mathrm{et}}^{1}\left(U, \mathbb{F}_{p}\right)=\operatorname{Hom}\left(\pi_{1}^{\mathrm{ab}}(U), \mathbb{F}_{p}\right)$, the vanishing of $\mathrm{H}_{\mathrm{et}}^{1}\left(U, \mathbb{F}_{p}\right)$ implies the assumption of Proposition 5. 
2.3. Using alterations. Section 2.2 reduces the proof of Theorem 2 in the presence of a big Cartier divisor to the following proposition.

Proposition 6. Let $k$ be an algebraically closed field and let $U / k$ be a connected normal variety with a big Cartier divisor and such that

(i) $\mathrm{H}^{0}\left(U, \mathscr{O}_{U}\right)=k$ and

(ii) there is a prime number $\ell$ such that $\operatorname{Pic}(U)$ is $\ell$-divisible.

Then $U$ has dimension 0.

Proof. By [de Jong 1996, Theorem 7.3], there exists an alteration, that is, a generically finite projective map $h: \tilde{U} \rightarrow U$ such that $\tilde{U}$ can be embedded into a connected smooth projective variety $\tilde{X}$.

$\underline{\text { Step } 1 .}$. The maximal open $V \subset U$, such that the restriction $\tilde{V}=h^{-1}(V) \rightarrow V$ is a finite map, has boundary $U \backslash V$ of codimension at least 2, since $U$ is normal.

The $k$-algebra $\mathrm{H}^{0}\left(\tilde{V}, \sigma_{\tilde{V}}\right)$ is an integral domain inside the function field of $\tilde{V}$. The minimal polynomial for $s \in \mathrm{H}^{0}\left(\tilde{V}, \mathscr{O}_{\tilde{V}}\right)$ with respect to the function field of $V$ has coefficients that are regular functions on $V$ by normality and uniqueness of the minimal polynomial. Hence these coefficients are elements of $\mathrm{H}^{0}\left(V, \mathrm{O}_{V}\right)=$ $\mathrm{H}^{0}\left(U, \mathrm{O}_{U}\right)=k$, and so

$$
\mathrm{H}^{0}\left(\tilde{V}, \mathrm{O}_{\tilde{V}}\right)=k
$$

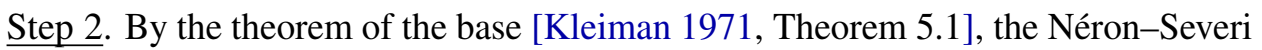
group

$$
\mathrm{NS}(\tilde{X})=\operatorname{Pic}(\tilde{X}) / \operatorname{Pic}^{0}(\tilde{X})
$$

is a finitely generated abelian group. Since the restriction map $\operatorname{Pic}(\tilde{X}) \rightarrow \operatorname{Pic}(\tilde{U})$ is surjective, the induced composite map

$$
h^{*}: \operatorname{Pic}(U) \rightarrow \operatorname{coker}\left(\operatorname{Pic}^{0}(\tilde{X}) \rightarrow \operatorname{Pic}(\tilde{U})\right)
$$

maps an $\ell$-divisible group to a finitely generated abelian group, and hence has finite image.

$\underline{\text { Step 3 }}$. Let $D$ be a big Cartier divisor on $U$. Since $h: \tilde{U} \rightarrow U$ is generically finite, the divisor $h^{*} D$ is also a big Cartier divisor. Moreover, as in the proof of Proposition 4 , there is a big divisor $\tilde{D}$ on $\tilde{X}$ that restricts to $h^{*} D$ on $\tilde{U}$. Upon replacing $D$ and $\tilde{D}$ by a positive multiple, we may assume, by the finiteness of the image of the map (2-1), that $\tilde{D}$ is algebraically and thus numerically equivalent to a divisor $B$ on $\tilde{X}$ that is supported in $\tilde{X} \backslash \tilde{U}$.

Since bigness on projective varieties only depends on the numerical equivalence class, see [Lazarsfeld 2004, Corollary 2.2.8], the divisor $B$ is also big. Restriction to $\tilde{V}$ yields

$$
\bigcup_{n \geq 0} \mathrm{H}^{0}\left(\tilde{X}, \mathcal{O}_{\tilde{X}}(n B)\right) \subseteq \mathrm{H}^{0}\left(\tilde{V}, \mathcal{O}_{\tilde{V}}\right)=k,
$$


by Step 1 above. We conclude that $\operatorname{dim} U=\operatorname{dim} \tilde{X}=0$ by the bigness of $B$.

2.4. Complementing example. We illustrate the importance of the presence of a big divisor in Theorem 2 or Proposition 6 by an example from toric geometry.

We first recall two facts about complete toric varieties that are standard analytically over $\mathbb{C}$ and that have étale counterparts for toric varieties over arbitrary algebraically closed base fields, in particular of characteristic $p>0$.

Lemma 7. Let $k$ be an algebraically closed field. Any complete toric variety $X / k$ is étale simply connected: $\pi_{1}(X)=\mathbf{1}$.

Proof. By toric resolution, see [Fulton 1993, §2.6], there is a resolution of singularities $\tilde{X} \rightarrow X$ with a smooth projective toric variety $\tilde{X}$. Birational invariance of the étale fundamental group shows $\pi_{1}(\tilde{X})=\pi_{1}\left(\mathbb{P}_{k}^{n}\right)=\mathbf{1}$, and the surjection $\pi_{1}(\tilde{X}) \rightarrow \pi_{1}(X)$ shows that $X$ is étale 1-connected.

Lemma 8. Let $k$ be an algebraically closed field of characteristic $p$, and let $X / k$ be a complete toric variety. Then for all $\ell \neq p$ we have

$$
\mathrm{H}_{\text {ét }}^{2}\left(X, \mathbb{Z}_{\ell}(1)\right) \simeq \operatorname{Pic}(X) \otimes \mathbb{Z}_{\ell} .
$$

Proof. In the context of toric varieties over $\mathbb{C}$ and with respect to singular cohomology, this is [Fulton 1993, Corollary in 3.4]. The $\ell$-adic case for toric varieties over an algebraically closed field $k$ of characteristic $\neq \ell$ follows with a parallel proof.

Example 9. Let $U=X$ be a complete normal nonprojective toric variety $X$ of dimension 3 with trivial Picard group. Such toric varieties have been constructed in [Eikelberg 1992, Example 3.5; Fulton 1993, pp. 25-26, 65]. These sources construct $X$ over $\mathbb{C}$ but the constructions work mutatis mutandis over any algebraically closed base field $k$. Then

(i) $\mathrm{H}_{\text {ét }}^{1}\left(X, \mathbb{F}_{p}\right)=0$ by Lemma 7 , and

(ii) $\mathrm{H}_{\text {et }}^{2}\left(X, \mathbb{Z}_{\ell}(1)\right)=0$ for all $\ell \neq p$ by Lemma 8 , and since there is nontrivial torsion in $\ell$-adic cohomology only for finitely many primes [Gabber 1983], we conclude that $\mathrm{H}^{2}\left(X, \mu_{\ell}\right)=0$ for almost all $\ell \neq p$.

Therefore the assumptions of Theorem 2 hold, with the exception of the presence of a big Cartier divisor. Nevertheless, these toric varieties are not étale contractible since $\mathrm{H}_{\mathrm{et}}^{6}\left(X, \mathbb{Z}_{\ell}(3)\right)=\mathbb{Z}_{\ell}$.

\section{Normal surfaces}

In this section, Proposition 10 completes the proof of Theorem 2 for surfaces. Not every normal surface admits a big Cartier divisor, so something needs to be done. Examples of proper normal surfaces with trivial Picard group, in particular without big divisors, can be found in [Nagata 1958; Schröer 1999]. However, on a 
hypothetical normal 2-contractible surface, a specialisation argument allows us to conclude the existence of a big Cartier divisor in general.

Proposition 10. There is no normal connected surface $U / k$ over an algebraically closed field $k$ of characteristic $p>0$ such that

(i) $\mathrm{H}_{\mathrm{et}}^{1}\left(U, \mathbb{F}_{p}\right)=0$ and

(ii) $\mathrm{H}_{\text {ét }}^{2}\left(U, \mu_{\ell}\right)=0$ for some prime number $\ell \neq p$.

Proof. We argue by contradiction and assume that $U$ is a surface as in the proposition. By Nagata's embedding theorem and resolution of singularities for surfaces, $U$ is a dense open in a normal proper surface $X / k$ with boundary $Y=X \backslash U$ being a normal crossing divisor. Hence, $X$ is smooth in a neighbourhood of $Y$.

By limit arguments, we may spread out over an integral scheme $S$ of finite type over $\mathbb{F}_{p}$, that is, there is a proper flat $f: \mathscr{X} \rightarrow S$, a relative Cartier divisor $\mathscr{Y}$ in $\mathscr{X} / S$ with normal crossing relative to $S$ and complement $\mathscr{U}=\mathscr{X} \backslash y$ such that

(a) all fibres are normal proper surfaces;

(b) there is a point $\eta: \operatorname{Spec}(k) \rightarrow S$ over the generic point of $S$ such that the fibre over $\eta$ agrees with the original $\mathscr{L}_{\eta}=X$ together with $\boldsymbol{U}_{\eta}=U$ and $\mathscr{Y}_{\eta}=Y$;

(c) the set of irreducible components of the fibres of 9 forms a constant system, and each component of $y$ is a Cartier divisor; and

(d) the higher direct image $\left.\mathrm{R}^{2} f\right|_{\varkappa_{*}} \mu_{\ell}$ is locally constant and commutes with arbitrary base change by [Deligne 1977, Finitude, Theorem 1.9].

Since the generic stalk $\left(\left.\mathrm{R}^{2} f\right|_{\iota_{*}} \mu_{\ell}\right)_{\eta}=\mathrm{H}_{\mathrm{ét}}^{2}\left(U, \mu_{\ell}\right)=0$ vanishes, we conclude that for all geometric points $\bar{s} \in S$, we have $\mathrm{H}_{\text {ét }}^{2}\left(U_{\bar{s}}, \mu_{\ell}\right)=0$, where $U_{\bar{s}}$ is the fibre of $U \rightarrow S$ in $\bar{s}$. As in the proof of Proposition 6, this implies that for every Cartier divisor $D$ on $\mathscr{X}_{\bar{s}}$, there are an $m \geq 1$ and a Cartier divisor $E$ on $\mathscr{X}_{\bar{s}}$ supported in $\mathscr{Y}_{\bar{s}}$ such that $m D \equiv E$ are numerically equivalent.

We apply this insight to a geometric fibre $\mathscr{X}_{\bar{t}}$ above a closed point $t \in S$. Since by [Artin 1962, Corollary 2.11], all proper normal surfaces over the algebraic closure of a finite field are projective, we conclude that there is a very ample Cartier divisor $H_{\bar{t}}$ on $\mathscr{X}_{\bar{t}}$ with support contained in $\mathscr{Y}_{\bar{t}}$.

Let $\mathscr{H} \hookrightarrow \mathscr{X}$ be the relative Cartier divisor with support in $\mathscr{Y}$ that specialises to $H_{\bar{t}}$. By [Grothendieck 1961, Théorème 4.7.1], the divisor $\mathscr{H}$ is ample relative to $S$ in an open neighbourhood of $t \in S$. Consequently, the normal proper surface $X$ is projective, and in particular, $U$ admits a big divisor. The part of Theorem 2 proven in Section 2.3 leads to a contradiction.

Remark 11. It follows from the proof of Proposition 10 that any proper nonprojective normal surface $X$ with trivial Picard group, in particular the examples of [Nagata 1958; Schröer 1999], must have $\mathrm{H}_{\text {êt }}^{2}\left(X, \mu_{\ell}\right) \neq 0$ and a fortiori must contain 
nontrivial $\ell$-torsion classes in the cohomological Brauer group $\operatorname{Br}(X)$ for all $\ell$ different from the characteristic. The existence of nontrivial torsion classes in $\operatorname{Br}(X)$ under the above assumptions was proven by different methods in [Schröer 2001, proof of Theorem 4.1].

\section{Acknowledgments}

We would like to thank Alexander Schmidt and Malte Witte for comments on an earlier version of the paper.

\section{References}

[Artin 1962] M. Artin, "Some numerical criteria for contractability of curves on algebraic surfaces", Amer. J. Math. 84 (1962), 485-496. MR 26 \#3704 Zbl 0105.14404

[Artin and Mazur 1969] M. Artin and B. Mazur, Etale homotopy, Lecture Notes in Mathematics 100, Springer, Berlin, 1969. MR 39 \#6883 Zbl 0182.26001

[Asok and Doran 2007] A. Asok and B. Doran, "On unipotent quotients and some $\mathbb{A}^{1}$-contractible smooth schemes", Int. Math. Res. Pap. 2007:2 (2007), rpm005. MR 2008f:14061 Zbl 1157.14032

[Deligne 1977] P. Deligne, Cohomologie étale: Séminaire de Géométrie Algébrique du Bois-Marie (SGA $4 \frac{1}{5}$ ), Lecture Notes in Mathematics 569, Springer, Berlin, 1977. MR 57 \#3132 Zbl 0349.14008 [tom Dieck and Petrie 1990] T. tom Dieck and T. Petrie, "Contractible affine surfaces of Kodaira dimension one", Japan. J. Math. (N.S.) 16:1 (1990), 147-169. MR 91j:14027 Zbl 0721.14018

[Eikelberg 1992] M. Eikelberg, "The Picard group of a compact toric variety", Results Math. 22:1-2 (1992), 509-527. MR 93g:14060 Zbl 0786.14031

[Fulton 1993] W. Fulton, Introduction to toric varieties, Annals of Mathematics Studies 131, Princeton University Press, 1993. MR 94g:14028 Zbl 0813.14039

[Gabber 1983] O. Gabber, "Sur la torsion dans la cohomologie $l$-adique d'une variété", C. R. Acad. Sci. Paris Sér. I Math. 297:3 (1983), 179-182. MR 85f:14018 Zbl 0574.14019

[Grothendieck 1961] A. Grothendieck, "Éléments de géométrie algébrique, III: Étude cohomologique des faisceaux cohérents, I", Inst. Hautes Études Sci. Publ. Math. 11 (1961), 167. MR 36 \#177c Zbl 0118.36206

[de Jong 1996] A. J. de Jong, "Smoothness, semi-stability and alterations", Inst. Hautes Études Sci. Publ. Math. 83 (1996), 51-93. MR 98e:14011 Zbl 0916.14005

[Kleiman 1971] S. Kleiman, "Les théorèmes de finitude pour le foncteur de Picard: Exposé XIII", pp. 616-666 in Théorie des intersections et théorème de Riemann-Roch: Séminaire de Géométrie Algébrique du Bois-Marie 1966-1967 (SGA 6), edited by P. Berthelot et al., Lecture Notes in Mathematics 225, Springer, Berlin, 1971. MR 50 \#7133 Zbl 0227.14007

[Kraft 1996] H. Kraft, "Challenging problems on affine $n$-space”, pp. 295-317 in Séminaire Bourbaki 1994/95 (Exposé 802 No. 5), Astérisque 237, Société Mathématique de France, Paris, 1996. MR 97m:14042 Zbl 0892.14003

[Lazarsfeld 2004] R. Lazarsfeld, Positivity in algebraic geometry, I: Classical setting: Line bundles and linear series, Ergebnisse der Mathematik und ihrer Grenzgebiete (3) 48, Springer, Berlin, 2004. MR 2005k:14001a Zbl 1093.14501

[Nagata 1958] M. Nagata, "Existence theorems for nonprojective complete algebraic varieties", Illinois J. Math. 2 (1958), 490-498. MR 20 \#3875 Zbl 0081.37503 
[Ramanujam 1971] C. P. Ramanujam, "A topological characterisation of the affine plane as an algebraic variety”, Ann. of Math. (2) 94 (1971), 69-88. MR 44 \#4010 Zbl 0218.14021

[Schröer 1999] S. Schröer, “On non-projective normal surfaces”, Manuscripta Math. 100:3 (1999), 317-321. MR 2001c:14067 Zbl 0987.14031

[Schröer 2001] S. Schröer, "There are enough Azumaya algebras on surfaces", Math. Ann. 321:2 (2001), 439-454. MR 2002i:14023 Zbl 1053.14017

[Winkelmann 1990] J. Winkelmann, "On free holomorphic $\mathbf{C}$-actions on $\mathbf{C}^{n}$ and homogeneous Stein manifolds”, Math. Ann. 286:1-3 (1990), 593-612. MR 90k:32094 Zbl 0708.32004

[Zaŭdenberg 1999] M. Zaĭdenberg, "Exotic algebraic structures on affine spaces", Algebra i Analiz 11:5 (1999), 3-73. In Russian; translated in St. Petersburg Math. J. 11:5 (2000), 703-760. MR 2001d:14069 Zbl 1002.14020

Communicated by Barry Mazur

Received 2013-10-31 Revised 2013-12-03 Accepted 2014-01-22

holschbach@mathi.uni-heidelberg Mathematisches Institut, Universität Heidelberg, Im Neuenheimer Feld 288, 69120 Heidelberg, Germany

jschmidt@mathi.uni-heidelberg.de Mathematisches Institut, Universität Heidelberg, Im Neuenheimer Feld 288, 69120 Heidelberg, Germany

stix@math.uni-frankfurt.de Institut für Mathematik, Johann Wolfgang Goethe-Universität, Robert-Mayer-Strasse 6-8, 60325 Frankfurt am Main, Germany 


\section{Algebra \& Number Theory}

msp.org/ant

\section{EDITORS}

MANAGING EDITOR

Bjorn Poonen

Massachusetts Institute of Technology

Cambridge, USA

\author{
EDITORIAL BOARD CHAIR \\ David Eisenbud \\ University of California \\ Berkeley, USA
}

BOARD OF EDITORS

Georgia Benkart

Dave Benson

Richard E. Borcherds

John H. Coates

J-L. Colliot-Thélène

Brian D. Conrad

Hélène Esnault

Hubert Flenner

Edward Frenkel

Andrew Granville

Joseph Gubeladze

Roger Heath-Brown

Craig Huneke

Yujiro Kawamata

János Kollár

Yuri Manin

Barry Mazur

Philippe Michel

Susan Montgomery
University of Wisconsin, Madison, USA

University of Aberdeen, Scotland

University of California, Berkeley, USA

University of Cambridge, UK

CNRS, Université Paris-Sud, France

University of Michigan, USA

Freie Universität Berlin, Germany

Ruhr-Universität, Germany

University of California, Berkeley, USA

Université de Montréal, Canada

San Francisco State University, USA

Oxford University, UK

University of Virginia, USA

University of Tokyo, Japan

Princeton University, USA

Northwestern University, USA

Harvard University, USA

École Polytechnique Fédérale de Lausanne

University of Southern California, USA
Shigefumi Mori

Raman Parimala

Jonathan Pila

Anand Pillay

Victor Reiner

Peter Sarnak

Joseph H. Silverman

Michael Singer

Vasudevan Srinivas

J. Toby Stafford

Bernd Sturmfels

Richard Taylor

Ravi Vakil

Michel van den Bergh

Marie-France Vignéras

Kei-Ichi Watanabe

Efim Zelmanov

Shou-Wu Zhang
RIMS, Kyoto University, Japan

Emory University, USA

University of Oxford, UK

University of Notre Dame, USA

University of Minnesota, USA

Princeton University, USA

Brown University, USA

North Carolina State University, USA

Tata Inst. of Fund. Research, India

University of Michigan, USA

University of California, Berkeley, USA

Harvard University, USA

Stanford University, USA

Hasselt University, Belgium

Université Paris VII, France

Nihon University, Japan

University of California, San Diego, USA

Princeton University, USA

\section{PRODUCTION}

production@msp.org

Silvio Levy, Scientific Editor

See inside back cover or msp.org/ant for submission instructions.

The subscription price for 2014 is US $\$ 225 /$ year for the electronic version, and $\$ 400 /$ year ( $\$ 55$, if shipping outside the US) for print and electronic. Subscriptions, requests for back issues and changes of subscribers address should be sent to MSP.

Algebra \& Number Theory (ISSN 1944-7833 electronic, 1937-0652 printed) at Mathematical Sciences Publishers, 798 Evans Hall \#3840, c/o University of California, Berkeley, CA 94720-3840 is published continuously online. Periodical rate postage paid at Berkeley, CA 94704, and additional mailing offices.

ANT peer review and production are managed by EditFLOw ${ }^{\circledR}$ from Mathematical Sciences Publishers.

\section{PUBLISHED BY}

mathematical sciences publishers

nonprofit scientific publishing

http://msp.org/

(C) 2014 Mathematical Sciences Publishers 


\section{Algebra \& Number Theory}

Volume $8 \quad$ No. $4 \quad 2014$

The derived moduli space of stable sheaves

Kai Behrend, Ionut Ciocan-Fontanine, Junho Hwang and Michael Rose

Averages of the number of points on elliptic curves

Greg Martin, Paul Pollack and Ethan Smith

Noncrossed product bounds over Henselian fields

Timo Hanke, DanNy NeFtin and JaCk SONN

Yangians and quantizations of slices in the affine Grassmannian

Joel Kamnitzer, Ben Webster, Alex Weekes and Oded Yacobi

Equidistribution of values of linear forms on quadratic surfaces

OLIVER SARGENT

Posets, tensor products and Schur positivity

Vyjayanthi Chari, Ghislain Fourier and Daisuke SAGaKi

Parameterizing tropical curves I: Curves of genus zero and one

DAVID E. SPEYER

Pair correlation of angles between reciprocal geodesics on the modular surface

Florin P. Boca, Vicențiu Pașol, AleXandru A. Popa and AleXandru

ZAHARESCU

Étale contractible varieties in positive characteristic

ARMin HolschbaCh, JohanNes SCHMIDT and JaKob STIX 